\title{
Infrared photoluminescence of high In-content InN/InGaN multiple-quantum-wells
}

Article in Physica Status Solidi (A) Applications and Materials · January 2012

Impact Factor: $1.62 \cdot$ DOI: 10.1002/pssa.201100188

CITATIONS

2

9 authors, including:

\section{Lorenzo Rigutti}

Université de Rouen

82 PUBLICATIONS 935 CITATIONS

SEE PROFILE
READS

22

\section{Eva Monroy}

Atomic Energy and Alternative Energies Co...

372 PUBLICATIONS 5,964 CITATIONS

SEE PROFILE 


\title{
Infrared photoluminescence of high In-content InN/InGaN multiple-quantum-wells
}

\author{
Sirona Valdueza-Felip ${ }^{*, 1}$, Lorenzo Rigutti ${ }^{2}$, Fernando B. Naranjo ${ }^{1}$, Bertrand Lacroix ${ }^{3}$, Susana Fernández ${ }^{4}$, \\ Pierre Ruterana ${ }^{3}$, François H. Julien ${ }^{2}$, Miguel González-Herráez, and Eva Monroy ${ }^{5}$ \\ ${ }^{1}$ Electronics Department, University of Alcalá, Madrid-Barcelona Road, km 33.6, 28871 Alcalá de Henares, Spain \\ ${ }^{2}$ Institut d'Electronique Fondamentale, University of Paris Sud XI, UMR 8622 CNRS, 91405 Orsay, France \\ ${ }^{3}$ Centre de Recherche sur les Ions les Matériaux et la Photonique (CIMAP), UMR 6252, CNRS, ENSICAEN, CEA, UCBN, \\ 6 Boulevard Maréchal Juin, 14050 Caen Cedex 4, France \\ ${ }^{4}$ Departamento de Energías Renovables, Energía Solar Fotovoltaica, CIEMAT, Avda. Complutense 22, 28040 Madrid, Spain \\ ${ }^{5}$ CEA Grenoble, INAC/SP2M, 25 Rue des Martyrs, 38042 Grenoble Cedex 9, France
}

Received 25 May 2011, revised 1 July 2011, accepted 4 July 2011

Published online 21 November 2011

Keywords heterostructures, molecular beam epitaxy, nitrides, photoluminescence

*Corresponding author: e-mail sirona.valdueza@depeca.uah.es, Phone: +34 91885 6913, Fax: +34 918856591

We report on the thermal evolution of the photoluminescence (PL) from high In-content $\mathrm{InN} / \mathrm{In}_{0.9} \mathrm{Ga}_{0.1} \mathrm{~N}$ multiple-quantum wells (MQWs) synthesized by plasma-assisted molecular-beam epitaxy on GaN-on-sapphire templates. The structural quality and the well/barrier thickness uniformity in the MQW structure are assessed by X-ray diffraction and transmission electron microscopy measurements. PL results are compared with the luminescence from a $1-\mu \mathrm{m}$-thick $\mathrm{InN}$ reference sample. In both cases, the dominant low-temperature (5 K) PL emission peaks at $\sim 0.73 \mathrm{eV}$ with a full width at half maximum of $\sim 86 \mathrm{meV}$. The InN layer displays an S-shape evolution of the emission peak energy with temperature, explained in terms of carrier localization. A carrier localization energy of $\sim 12 \mathrm{meV}$ is estimated for the InN layer, in good agreement with the expected carrier concentration. In the case of the MQW structure, an enhancement of the carrier localization associated to the piezoelectric field results in an improved thermal stability of the PL intensity, reaching an internal quantum efficiency of $\sim 16 \%$.
1 Introduction InN has extended the operation wavelength of III-nitrides to the near-infrared (NIR) range $(\sim 0.65 \mathrm{eV}, 1.9 \mu \mathrm{m})[1]$ raising the possibility of new devices for application in photovoltaics, chemical sensing, high power/high speed electronics, high-efficiency optoelectronics, solid-state lighting, and terahertz emission and detection [2]. InN is also a promising material for all-optical signal processing applications in optical communication networks, which require new optical components for ultra fast pulse generation, dispersion and delay control ("slow light generation") [3], switching, preamplification and wavelength conversion at $1.55 \mu \mathrm{m}$ [4]. This new generation of efficient devices should be characterized by high-quality materials with low optical control power, high speed operation and improved working performance at room temperature. From this point of view, low-dimensional InN-based semiconductor structures such as InN multi-quantum-wells (MQWs) [5-9] or quantum dots (QDs) [10-13] can attain the required specifications taking advantage of the tunability of their operation wavelength through band-gap engineering. Furthermore, these heterostructures are attractive for photonic applications because they exhibit strong quantum confinement effects, which should lead to an enhanced linear and nonlinear response at resonant wavelengths. As an example, strong absorption saturation at $1.5 \mu \mathrm{m}$ has been recently demonstrated in InN/InGaN MQWs with high In content in the barriers [14]. In this work, we investigate the thermal evolution of the infrared photoluminescence (PL) of InNbased MQWs for its application to high-efficiency NIR optoelectronic devices.

Experimental details The analysed MQW structure consists of 41 periods of $4.5 \mathrm{~nm}$ InN wells embedded in $7 \mathrm{~nm}$ InGaN barriers with a nominal $10 \%$ of Ga composition. The structure was grown by plasma-assisted molecular-beam epitaxy (PAMBE) on 10- $\mu$ m-thick GaN-on-sapphire templates. The inset of Fig. 1 shows a schematic description of the MQW structure, which starts with InN at the template/ 


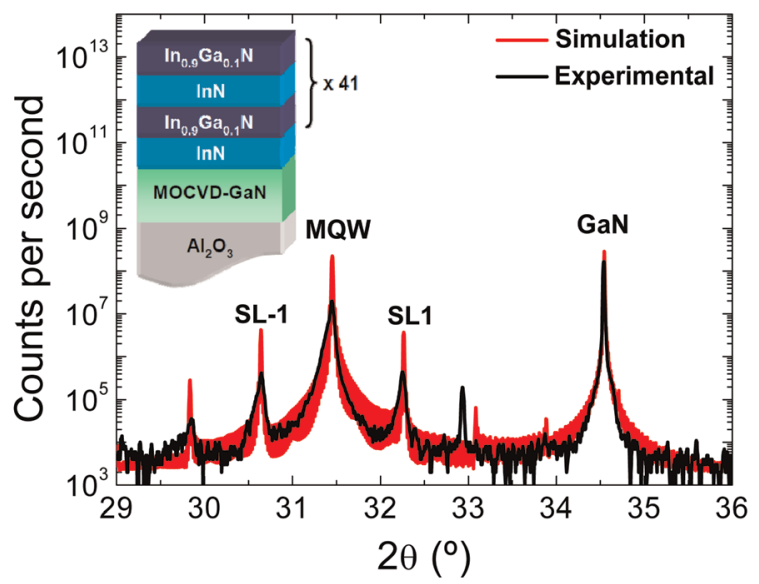

Figure 1 (online colour at: www.pss-a.com) HRXRD $\theta-2 \theta$ scan around the (0002) X-ray reflection of the $\mathrm{InN} / \mathrm{In}_{0.9} \mathrm{Ga}_{0.1} \mathrm{~N}$ MQW structure compared to theoretical calculations.

MQW interface in order to ensure the InN lattice parameter in the MQWs. The well and barrier thicknesses were designed to obtain an interband transition at $\sim 1.5 \mu \mathrm{m}$. A reference sample consisting of $1-\mu \mathrm{m}$-thick InN layer on top of an $\mathrm{InN} / \mathrm{In}_{0.7} \mathrm{Ga}_{0.3} \mathrm{~N}$ superlattice (SL) (11 periods) was grown on GaN-template substrates. The introduction of the SL as a buffer layer reduces the threading dislocation density and improves the optical performance of the subsequent $\mathrm{InN}$ film. Both samples were grown at $450{ }^{\circ} \mathrm{C}$, with a $\mathrm{N}$-limited growth rate of $280 \mathrm{~nm} / \mathrm{h}$.

The structural and morphological quality of the samples was assessed by high-resolution X-ray diffraction (HRXRD), transmission electron microscopy (TEM) and atomic force microscopy (AFM) measurements. Linear optical properties were studied through transmission measurements in the $730-2250 \mathrm{~nm}$ spectral range using a UV/VIS/NIR Perkin-Elmer Lambda 1050 scanning spectrometer. Temperature dependent $(5-300 \mathrm{~K})$ PL measurements were carried out by exciting with a continuous-wave $\operatorname{Ar}^{+}$laser $(\lambda=488 \mathrm{~nm})$ focused onto a $3-\mu \mathrm{m}$ diameter spot. The emission was collected with an InAs detector using lock-in techniques.

3 Results and discussion The crystalline structure of the samples was evaluated through HRXRD measurements. Figure 1 displays the $\theta-2 \theta$ scan around the (0002) $\mathrm{X}$-ray reflection of the MQW sample compared to theoretical calculations. The observation of several satellite peaks of the MQW reflection confirms the good periodicity of the structure. Furthermore, the well and barrier thickness of the MQWs was verified by fitting the X-ray diffraction spectrum to the simulation. The surface morphology was analysed by AFM measurements in areas of $2 \times 2 \mu \mathrm{m}^{2}$. Flat surfaces displaying atomic terraces and with a root-mean-square roughness, $\sim 0.5 \mathrm{~nm}$ were obtained for both $\mathrm{InN}$ and MQW samples, as illustrated in Fig. 2.
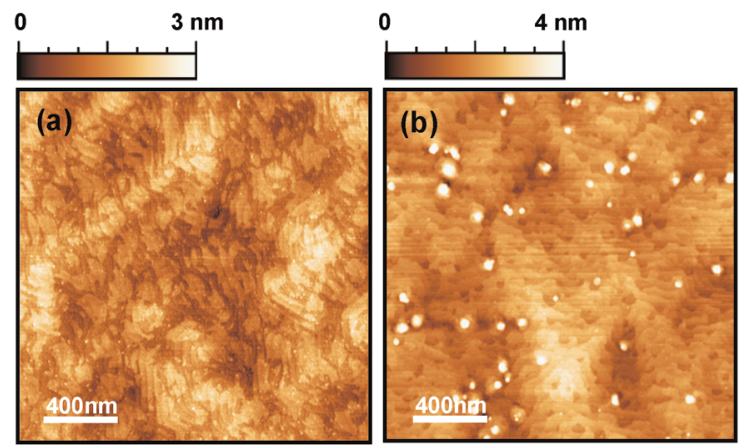

Figure 2 (online colour at: www.pss-a.com) AFM image of $2 \times 2 \mu \mathrm{m}^{2}$ area for the (a) $\mathrm{InN}$ bulk and (b) $\mathrm{InN} / \mathrm{In}_{0.9} \mathrm{Ga}_{0.1} \mathrm{MQW}$ samples.

A fine structural analysis of the samples was carried out by TEM measurements. The TEM analysis of the InN bulk sample shows that the SL buffer layer helps to reduce the threading dislocations, which are mostly of edge and mixed type [15]. The fluctuations in strain induced by the alternation of materials in the SL probably force many dislocations to bend, forming segments of misfit dislocations at the interfaces [16]. Moreover, they may react and annihilate when bending towards or back in the growth direction [17]. Indeed, no other types of defects such as partial dislocations or stacking faults were found in the layers [18], and no ordering was observed to take place in the InGaN layers, as previously reported for MBE and MOVPE growth $[19,20]$.

For the MQW sample, as illustrated in the TEM image of Fig. 3, the InN QWs are the bright areas and the InGaN barriers correspond to the dark ones. A good uniformity of the well and barrier thicknesses was obtained from the measured intensity profile [14], in agreement with results obtained from HRXRD measurements.

Regarding the optical properties, Fig. 4 shows the optical transmission spectra of the InN and the MQW samples. The observed absorption band edge blue shifts to higher energies in the MQW sample due to the transmission of the InGaN barriers.

Low-temperature $(T=5 \mathrm{~K})$ PL measurements reveal a dominant infrared PL emission peaking at $\sim 1.7 \mu \mathrm{m}(0.72$ $0.73 \mathrm{eV}$ ) for both $\mathrm{InN}$ and MQW samples, showing a similar

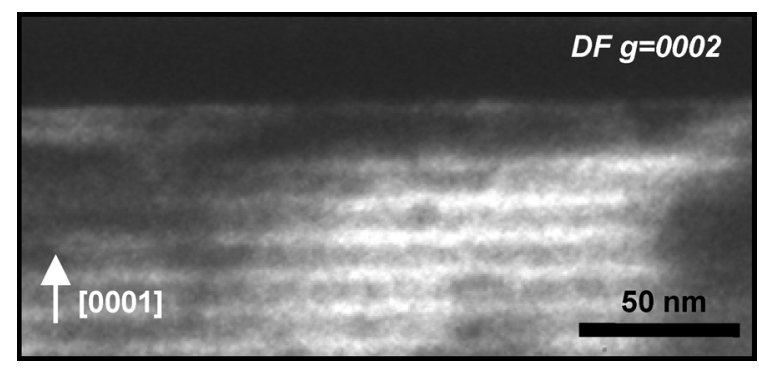

Figure 3 Cross-sectional dark field $(g=0002)$ TEM image of the $\mathrm{InN} / \mathrm{In}_{0.9} \mathrm{Ga}_{0.1} \mathrm{~N}$ MQW sample. 


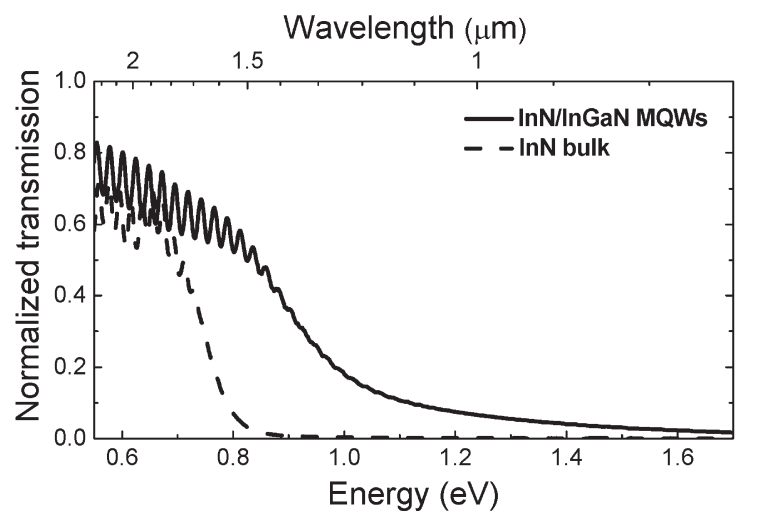

Figure 4 Room-temperature normal incidence transmission measurements of the InN bulk and the MQW sample.

broadening of the emission with a FWHM of $\sim 86 \mathrm{meV}$. In order to investigate the origin of the luminescence, the thermal evolution of the infrared PL peak energy was studied between $5 \mathrm{~K}$ and room temperature. The emission from the InN layer displays a strong S-shape evolution with temperature, as illustrated in Fig. 5. This S-shape behaviour begins with a blue shift of the emission energy at low temperatures $(5-125 \mathrm{~K})$, which is attributed to the localization of carriers in local potential minima caused by defectrelated inhomogeneities, as observed by other authors [21].

For temperatures higher than $125 \mathrm{~K}$, the PL red shifts following Varshni's equation [22]

$$
E_{\mathrm{g}}(T)=E_{\mathrm{g}}(0)-\gamma \frac{T^{2}}{\beta+T},
$$

where $E_{\mathrm{g}}(0)$ is the PL emission energy at $0 \mathrm{~K}, \gamma$ is a proportionality constant and $\beta$ is physically associated with the Debye temperature of the crystal [22]. For the temperature dependent PL measurements plotted in the inset of Fig. 5, the best fit is obtained for $E_{\mathrm{g}}(0)=0.733 \mathrm{eV}$,

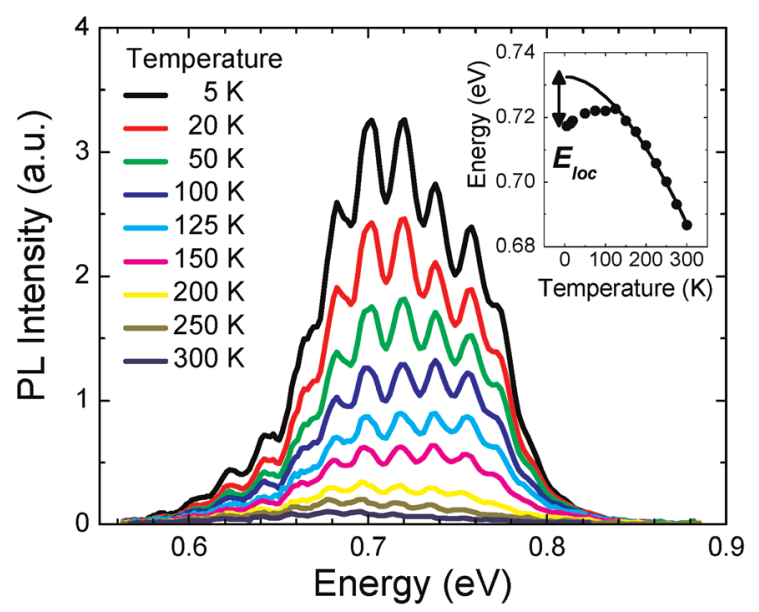

Figure 5 (online colour at: www.pss-a.com) PL spectra of the InN bulk sample as a function of temperature. Inset: thermal evolution of the emission peak energy.

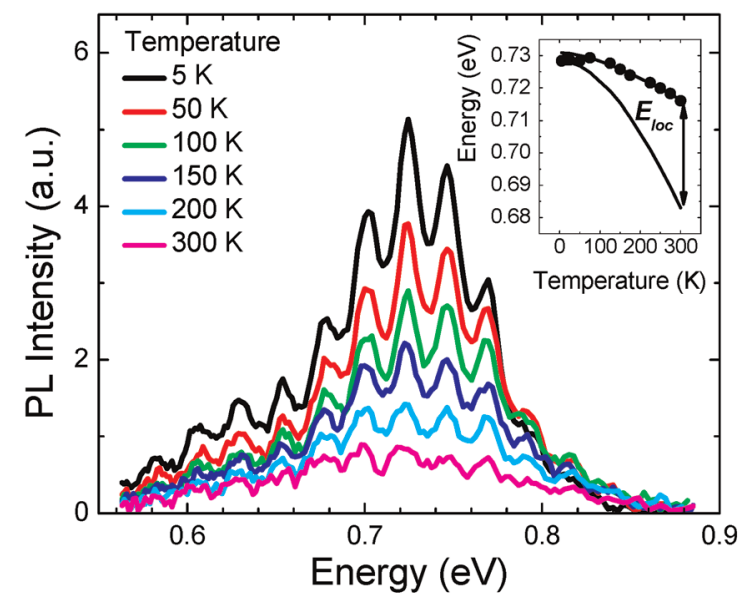

Figure 6 (online colour at: www.pss-a.com) Thermal evolution of the luminescence of the MQW sample. Inset: emission peak energy versus temperature.

$\gamma=0.59 \mathrm{meV} / \mathrm{K}$ and $\beta=869 \mathrm{~K}$; with $E_{\mathrm{g}}(5 \mathrm{~K})-E_{\mathrm{g}}(300 \mathrm{~K})$ $\sim 31 \mathrm{meV}$. These values are in good agreement with the ones previously reported by Wu et al. [23] $(\gamma=0.481 \mathrm{meV} / \mathrm{K}$ and $\beta=865 \mathrm{~K}$ ) for InN samples deposited on sapphire by PAMBE with similar carrier concentrations. As illustrated in the inset of Fig. 5, from the difference between the expected PL emission energy at low temperature $\left(E_{\mathrm{g}}(0)\right.$ from Varshni's equation) and the experimental value, we estimate a localization energy $E_{\mathrm{loc}} \sim 12 \pm 2 \mathrm{meV}$, in agreement with the expected carrier localization of the sample in the range of $\sim 10^{19} / \mathrm{cm}^{3}$ [24].

Figure 6 shows the thermal evolution of the PL emission of the MQW sample. A strong deviation of the PL energy emission with temperature compared to the InN bulk sample and Varshni's equation is observed, which points to a carrier localization energy larger than $35 \mathrm{meV}$. This enhancement of carrier localization can be attributed to fluctuations of layer thickness and alloy composition in the barriers, whose effect is increased by the intense polarization field in InGaN alloys. This carrier confinement in these fluctuations screen nonradiative recombination centres, thus resulting in an improvement of the thermal stability of the PL of the InNbased MQWs in comparison to the InN layer. The behaviour is quite similar to the one observed in InGaN/GaN QD structures, due to the three-dimensional carrier confinement [25]. Note that the internal quantum efficiency (defined as the ratio between the luminescence at room temperature and at low temperature) of the MQW structure is around $16 \%$, to be compared with $\sim 3 \%$ for the InN layer.

4 Conclusions We have studied the thermal evolution of the PL emission in $\mathrm{InN} / \mathrm{In}_{0.9} \mathrm{Ga}_{0.1} \mathrm{~N}$ MQWs, displaying room temperature PL emission at $1.7 \mu \mathrm{m}$. A 1 - $\mu \mathrm{m}$-thick InN layer is also analysed for comparison. In the $\mathrm{InN}$ layer, an S-shape evolution of the PL emission energy with temperature is observed, attributed to carrier localization effects with a localization energy $\sim 12 \mathrm{meV}$. In the case of the 
MQWs, the localization energy increases above $35 \mathrm{meV}$, which is attributed to in-plane fluctuations of the electronic potential in the quantum well due to variation of the layer thickness and alloy fluctuations in the barriers. This localization results in an enhanced thermal stability of the $\mathrm{PL}$ in InN/InGaN MQW structures.

Acknowledgements Partial financial support was provided by Spanish Government Projects, TEC2009-14423-C02-02, by Comunidad de Madrid Project by No. S2009/ESP-1781.

\section{References}

[1] J. Wu, W. Walukiewicz, W. Shan, K. M. Yu, J. W. Ager, S. X. Li, E. E. Haller, H. Lu, and W. J. Schaff, J. Appl. Phys. 94, 4457 (2003).

[2] J. Wu, J. Appl. Phys. 106, 011101 (2009).

[3] F. B. Naranjo, M. González-Herráez, S. Valdueza-Felip, H. Fernández, J. Solis, S. Fernández, E. Monroy, J. Grandal, and M. A. Sánchez-García, Microelectron. J. 40, 349 (2009).

[4] O. Wada, New J. Phys. 6, 183 (2004).

[5] S. B. Che, W. Terashima, Y. Ishitani, A. Yoshikawa, T. Matsuda, H. Ishii, and S. Yoshida, Appl. Phys. Lett. 86, 261903 (2005).

[6] A. Yoshikawa, S. B. Che, W. Yamaguchi, H. Saito, X. Q. Wang, Y. Ishitani, and E. S. Hwang, Appl. Phys. Lett. 90, 073101 (2007).

[7] M. Kurouchi, H. Na, H. Naoi, D. Muto, S. Takado, T. Araki, T. Miyajima, and Y. Nanishi, Phys. Status Solidi C 3, 1599 (2006).

[8] S. Hirano, T. Inoue, G. Shikata, M. Orihara, Y. Hijikata, H. Yaguchi, and S. Yoshida, J. Cryst. Growth 301/302, 513 (2007).

[9] J. Grandal, J. Pereiro, A. Bengoechea-Encabo, S. FernándezGarrido, M. A. Sánchez-García, E. Muñoz, E. Calleja, E. Luna, and A. Trampert, Appl. Phys. Lett. 98, 061901 (2011).

[10] W. C. Ke, C. P. Fu, C. Y. Chen, L. Lee, C. S. Ku, W. C. Chou, W. H. Chang, M. C. Lee, W. K. Chen, W. J. Lin, and Y. C. Cheng, Appl. Phys. Lett. 88, 191913 (2006).
[11] L. Zhou, T. Xu, D. J. Smith, and T. D. Moustakas, Appl. Phys. Lett. 88, 231906 (2006).

[12] J. Y. Chen, G. C. Chi, P. J. Huang, M. Y. Chen, S. C. Hung, C. H. Nien, M. C. Chen, S. M. Lan, B. J. Pong, C. J. Pan, C. J. Tun, F. Ren, C. Y. Chang, and S. J. Pearton, Appl. Phys. Lett. 92, 162103 (2008).

[13] M. Kumar, B. Roul, T. N. Bhat, M. K. Rajpalke, N. Sinha, A. T. Kalghatgi, and S. B. Krupanidhi, Adv. Sci. Lett. 3, 379 (2010).

[14] F. B. Naranjo, P. K. Kandaswamy, S. Valdueza-Felip, V. Calvo, M. González-Herráez, S. Martín-López, P. Corredera, J. A. Méndez, G. R. Mutta, B. Lacroix, P. Ruterana, and E. Monroy, Appl. Phys. Lett. 98, 031902 (2011).

[15] V. Potin, P. Ruterana, G. Nouet, R. C. Pond, and H. Morkoç, Phys. Rev. B 61, 5587 (2000).

[16] P. Khomninou, T. Kehagias, G. Nouet, P. Ruterana, and T. Karakostas, Phys. Rev. B 64, 195329 (2001).

[17] P. K. Kandaswamy, C. Bougerol, D. Jalabert, P. Ruterana, and E. Monroy, J. Appl. Phys. 106, 013526 (2009).

[18] V. Potin, P. Ruterana, and G. Nouet, J. Phys.: Condens. Matter 12, (1030). 1(2 000).

[19] D. Doppalapudi, S. N. Basu, and T. D. Moustakas, J. Appl. Phys. 85, 883 (1999).

[20] P. Ruterana, G. Nouet, W. Van der Stricht, I. Moerman, and L. Considine, Appl. Phys. Lett. 72, 1742 (1998).

[21] Y.-H. Cho, G. H. Gainer, A. J. Fischer, J. J. Song, S. Keller, U. K. Mishra, and S. P. DenBaars, Appl. Phys. Lett. 73, 1370 (1998).

[22] Y. P. Varshni, Physica 34, 149 (1967).

[23] J. Wu, W. Walukiewicz, W. Shan, K. M. Yu, J. W. Ager, III S. X. Li, E. E. Haller, Hai. Lu, and W. J. Schaff, J. Appl. Phys. 94, 4457 (2003).

[24] B. Liu, Z. Zhang, R. Zhang, D. Y. Fu, Z. L. Xie, H. Lu, W. J. Schaff, L. H. Song, Y. C. Cui, X. M. Hua, P. Han, Y. D. Zheng, Y. H. Chen, and Z. G. Wang, Appl. Phys. A 99, 139 (2010).

[25] Ž. Gačević, A. Das, J. Teubert, Y. Kotsar, P. K. Kandaswamy, Th. Kehagias, T. Koukoula, Ph. Komninou, and E. Monroy, J. Appl. Phys. 109, 103501 (2011). 